\title{
Computer Simulation of Spacecraft Onboard Equipment
}

\author{
L. Nozhenkova, O. Isaeva, E. Gruzenko \\ Institute of computational modelling SB RAS \\ Russia
}

\begin{abstract}
This article presents the results of application of the computer simulation technology for intellectual support of spacecraft onboard equipment design. Methods and instruments allowing construction of informational and graphical models were developed describing architecture of onboard systems and configuration of switching connections. Knowledge base creation and application means allow to specify logics of the model functioning, carry out simulation experiments and analyze the results of modeling. The program complex "Program and mathematical model of a spacecraft command-measuring system's onboard equipment" was developed to unite instruments of simulation modeling and intellectual support of onboard equipment design.
\end{abstract}

Keywords-c spacecraft; onboard equipment; command and measuring system; program and mathematical model; simulation modeling

\section{INTRODUCTION}

System analysis and computer simulation technologies are an integral part of modern scientific-and-technical activity. European Space Agency standards specify system development as an interdisciplinary approach managing all the processes of transformation of technical requirements into system solutions. It is also the basis of space industry development [1]. This article describes application of system engineering and computer simulation for spacecraft command-measuring system's onboard equipment construction support. Commandmeasuring system is a complex technical object basing on hightech elements and software. Onboard systems' experimental research meets economical and technological hardships due to complicity and variety of their functions and purposes [2]. In spite of the fact that computer simulation is a simple and clear form of complex technical objects' analysis, it hasn't been implemented properly in the space industry [3] due to high requirements to any applied technologies, complexity of usage of the existing program solutions, the necessity to have highly qualified and experienced staff. As a rule, the existing software is designed to optimize the structure, range and placement of equipment allowing to find the decision which meets the given requirements for mass-and-size parameters, physical characteristics and usage conditions [4]. Such approach is not enough to model the objects having their own functioning logics. Construction program support requires creation of modeling techniques in order to carry out simulation tests to research the work of equipment in different conditions. In this case, modeling helps to understand properties of the studied object due to general simplification or separating the model into simpler parts [5]. An obligatory requirement for development is a simple and clear software.

The program complex "Program and mathematical model of spacecraft command-measuring system's onboard equipment" was developed to complete the tasks of modeling. The complex contains instruments for graphic modeling of the equipment's architecture and configuration, instruments for intellectual support of construction and simulation of modeling software.

\section{INFORMATION-AND-GRAPHIC MODEL CONSTRUCTION}

The command-measuring system is one of the central systems of a spacecraft. Its structure and functions are determined by its purpose. It is designed for command-andprogram control of onboard systems, for transmission of telemetric information to earth systems, for measurement of current orbit navigation parameters, etc. Informational interaction with a spacecraft is fulfilled with the help of two data types: telecommands and telemetry. Structure and methods of data transmission are regulated by the European Space Agency standards. Telecommands are transmitted from an Earth flight control complex to a spacecraft on the basis of ESAPSS-04-107 standard [6]. Telemetry is a data flow transmitted from a spacecraft to the Earth in accordance with the ESAPSS-04-106 standard [7].

Graphic modeling instruments allow to simply and clearly describe the hardware of a command-measuring system. Work with the software includes visual placement, model blocks' configuration setting and setting of commutation interfaces, connections and parameters of the modelled equipment.

To support the functions of information-and-graphic modeling there exists a base configuration of the main units receiver, transmitter, interface module of the commandmeasuring system, onboard digital computer complex, onboard control complex, remote signaling etc. The instruments allow to build arbitrary models using base elements, and also to set user structures. A simple example of information-and-graphic model is presented in fig. 1, where: OCS CU - onboard control complex, CCU - command-measuring system's interface module, ODGS - onboard remote signaling equipment. Lines between the graphical elements show directions of information exchange during command transmission and telemetry. 




FIGURE I. AN INFORMATION-AND-GRAPHIC MODEL

The model contains a set of parameters and characteristics that can be changed configuring different variants of spaceship command-measuring system's onboard equipment. Visual building of a model with graphical elements gives significant advantages due to simplicity and clarity of onboard equipment projecting, analysis and tuning of the designed solutions [8].

\section{MODELING OF ONBOARD EQUIPMENT FUNCTIONS}

Computer simulation of different ways of functioning of spacecraft command-measuring system's onboard equipment is fulfilled with the help of the original instruments of the construction intellectual support. They allow to formally describe different variants of interaction between graphical model's elements in order to simulate real equipment's behavior.

Spacecraft command-measuring system onboard equipment's function scheme can be described as several key sequences of actions. Command-measuring system's interface module sends periodical requests to onboard remote signaling equipment in order to get telemetric information. Onboard remote signaling equipment processes an requests and, if it's correct, sends back a package containing board's telemetry. On the basis of the telemetric information acquired from onboard remote signaling equipment and from command-measuring system, the interface module forms telemetric package in accordance with the ESAPSS-04-106 standard [7] and sends it to the transmitter. The transmitter, in its turn, sends the package to Earth control complex. The Earth control complex receives packages of telemetric information. In order to complete command-measuring control, it forms and telecommands packages in accordance with the PSS-04-107 standard [6]. The receiver accepts packages and sends them to commandmeasuring system's interface module, which, in its turn, processes the commands and produces proper responses depending on their purposes. The commands control the choice of main or redundant equipment, the types of active commutation interfaces for onboard systems' interaction, data transmission speed, equipment's modes of functioning. For every action there exist the parameters of delay, waiting of response, repeated enquiries and switching to reserve communication channels, etc. In addition, actions to manage exceptional situations are included.
There are no unambiguous and strictly defined algorithms of onboard equipment functioning, in different cases a designer needs to consider different ways to realize its functions. In order to support construction and to provide simple and clear methods for description of the logics of work of commandmeasuring system, in this article we apply methods of artificial intelligence. There created a data base containing model elements' function rules. Software tools for its support and replenishment were created as well. Such approach is widely applied in different systems of engineering and technical constriction. It allows not only to get quality solutions, but also to exchange knowledge between groups of engineers highly experienced in different aspects of the problems solved [9]. In this case the data base is a storage of functional and technological processes allowing not only to watch, but also to control the process of inference [8].

The data base [10] consists of the condition-action rules «If A then B». In the right part, the rules contain actions for changing model's condition, and in the left part - the conditions under which they are executed. The data base is structured in accordance with the types of the elements and the scope of the rules' action. Fig. 2 shows a part of the data base describing the methods of spacecraft onboard commandmeasuring systems' functioning.

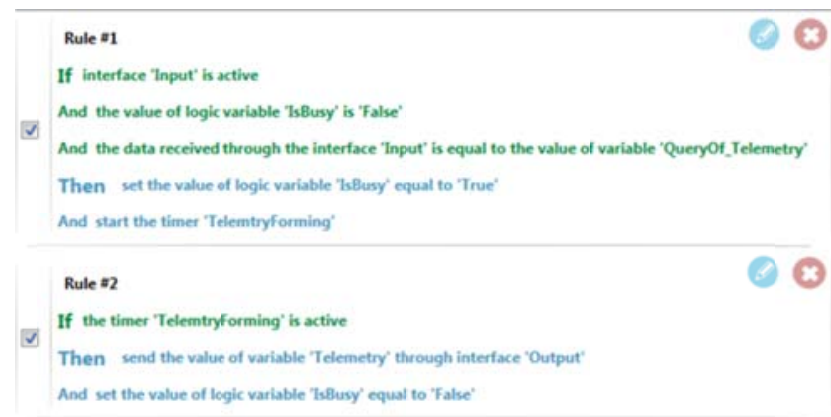

FIGURE II. A PART OF THE DATA BASE

There exist the general rules, rules of model elements' functioning, rules of command and command-and-program information transfer to the spacecraft systems, rules of telemetric requests, rules of onboard equipment's condition control, rules of setting of modes and interfaces for equipment functioning, etc. The structure and presentation form of knowledge allow an engineer to manipulate graphical elements and information characteristics of the onboard equipment model in order to complete different simulation tests.

\section{COMPLETION OF Simulation TESTS}

There are software means that allow, using an informationgraphical model, to simulate processes which take place in the command-measuring system. Simulation of a model functioning is performed by step-by-step or automatic change of its condition in accordance with the rules given in data base. For this purpose, in the process of inference [10] the rules applicable to a current state of the model are chosen, actions are performed and command execution is displayed, forming and transmission of data packages is simulated. Simulation experiments help to understand the system deeply [5], they give an idea of how it responds to external influences, emergency 
situation and how it develops through time. Software simulates and visualizes changes in condition and activity of model's elements, forming and transmission of data packages through commutation interfaces. Graphically, every data transmission is visualized by animation effect $s$. Interactive process of modeling allows the designer to carry out simulation tests by changing the parameters of signal reception and transmission, waiting time for responses and receipts, by switching active commutation interfaces and elements of the model in order to analyze its behavior during emergency situations.

An important instrument for understanding and analysis of simulation tests is the modeling protocol, fig. 3 .

\begin{tabular}{|c|c|c|c|c|}
\hline Transmitter - & & & & \\
\hline Transmission start time & Duration of transmission (ms) & Iransmitter intert- & Peceiver & Recever interface \\
\hline \multicolumn{5}{|l|}{ (1) Transmitter: $\mathrm{CCU}$} \\
\hline $00.00 \cdot 000100000$ & & 1 To ODGS (RS-232) & 0005 & Input (RS-232) \\
\hline $00,0000,4100000$ & & 1 To 0005 (RS-232) & ODGS & Input (PS-232) \\
\hline 00.0000 .8100000 & & 1 To ODGS (RS-232) & 0005 & Input (75-232) \\
\hline 0000.012100000 & & 1 To ODOS (RS-232) & 0005 & Input (RS-232) \\
\hline 00.00 .01 .6100000 & & 1 To ODGS (RS-232) & oDGs & Input (R5-232) \\
\hline \multicolumn{5}{|l|}{ Q Transmitter: ODGS } \\
\hline 0000000.0300000 & 43 & 3 Output (RS-232) & $\ll C U$ & From ODGS (RS.... \\
\hline 00.0000 .4300000 & 43 & 3 Output (RS-232) & $\mathrm{CCU}$ & From ODES (RS.- \\
\hline $00.00 \cdot 00.8300000$ & 43 & 3 Output (RS-232) & CCU & From ODGS (RS... . \\
\hline
\end{tabular}

FIGURE III. AN EXAMPLE OF A SIMULATION MODELING PROTOCOL

Protocol are presented as tables allowing to look through data and detail it, to group the events, to identify receiving and transmitting data sources. They allow to analyze sequence of the completed actions, amount and structure of the sent data, follow changes of the parameters and characteristics, identify reactions to the events happening in the model. Such approach allows the designer to study how and why the system performs different actions, thus understanding how the model needs to be changed in order to achieve the required function.

\section{CONCLUSION}

Creation of a full-functional version of the program complex "Program and mathematical model of spacecraft command-measuring system's onboard equipment" is scheduled in year 2015. Presently, it is being tested and got positive reviews from the space system design company. The work is financially supported by the Ministry of Education and Science of Russian Federation, and is performed by the Institute of Computational Modeling of the Siberian Branch of the Russian Academy of Science (contract № 02.G25.31.0041).

Application of the methods of computational modeling will help to reduce the cost of command-measuring systems' design, to shorten the production cycle and to reach high quality output. Software, in this case, is an intellectual partner of a designer, helping to carry out simulation experiments in order to build and analyze engineering solutions.

\section{REFERENCES}

[1] Space engineering. System engineering general requirements (ECSS-EST-10C), European space agency (ESA): ESTEC, 100 p., 2009.

[2] Vasile, M. \& Minisci, E. \& et al. Fast evidence-based space system engineering, 62nd International Astronautical Congress: South Africa, 2011.
[3] Guo, J Incorporating Multidisciplinary Design Optimization into Spacecraft Systems Engineering, 8th Conference on Systems Engineering Research: Hoboken, NJ, March 17-19, 2010.

[4] Higdon, K.P. \& Klaus D.M. Multidisciplinary design optimization for the development of human spacecraft, Earth \& Space: American Society of Civil Engineers, 2008.

[5] System Design, Modeling, and Simulation using Ptolemy II: Ptolemy.org, 2014.

[6] Packet Telecommand Standard (ESA PSS-04-107) Issue 2: European space agency, 166 p., 1992.

[7] Packet Telemetry Standard (ESA PSS-04-106) Issue 1: European space agency, 73 p., 1988.

[8] Stanley, G.M., Experiences using knowledge-based reasoning in online control systems, International Federation of Automatic Control (IFAC) Symposium on Computer Aided Design in Control Systems, July 15-17, 1991, Swansea, UK.

[9] Hernandez, J.A. \& Peters, T.J. and et al., Intelligent Decision Support for Assembly System Design, The Third Conference on Innovative Applications of Artificial Intelligence: The AAAI Press, Menlo Park, California, 1991.

[10] Russell \& Stuart, J. Artificial intelligence: a modern approach, PrenticeHall, Inc. A Simon \& Schuster Company Englewood Cliffs: New Jersey, 932 p., 1995. 